\title{
Clinical significance of EBP50 overexpression assessed by quantum dot analysis in gastric cancer
}

\author{
XIAO-GUANG LV, XIAO-FEI LEI, MENG-YAO JI, XU-FENG GUO, JING WANG and WEI-GUO DONG \\ Department of Gastroenterology, Renmin Hospital of Wuhan University, Wuhan, Hubei 430060, P.R. China
}

Received December 5, 2012; Accepted March 1, 2013

DOI: $10.3892 / 01.2013 .1271$

\begin{abstract}
Ezrin-radixin-moesin-binding phosphoprotein 50 (EBP50) is a postsynaptic density-95/disc-large/zonula occludens-1 (PDZ) homologous domain-containing protein that is involved in cell signaling. EBP50 regulates cell apoptosis, proliferation and invasion. In the present study, the prognostic impact factor of EBP50 expression was evaluated using a quantum dot (QD)-based assay and immunohistochemistry (IHC). The EBP50 protein expression in gastric cancer (GC) tissues was evaluated using IHC and QD-IHC. The study included 101 patients with GC (29 females and 72 males, aged 24-81 years), diagnosed and treated at the General Surgery Department of Renmin Hospital of Wuhan University (Wuhan, China) between 2000 and 2005. The survival rate was calculated using the Kaplan-Meier method and log-rank tests. IHC and QD analyses of $101 \mathrm{GC}$ tissue specimens revealed that EBP50-positive tumor cells were frequently present in GC. Increased EBP50 immunostaining was observed in 63 specimens (62.4\%). The EBP50 expression levels were correlated with increased tumor size and the male gender. EBP50 was well distributed in the cytoplasm and nuclei of the GC cells. However, EBP50 protein expression exhibited no correlation with age, differentiation, stage or lymph node metastasis. There were no associations between the expression of EBP50 and the mean survival rates (IHC, 50.5 vs. 58.1 months, $\mathrm{P}>0.05$; QD, 55.4 vs. 63.2 months, $\mathrm{P}>0.05)$. These findings suggest that EBP50 protein expression is not correlated with the prognosis of patients with GC. QD-IHC and IHC have similar advantages for the detection of EBP50 protein expression.
\end{abstract}

\section{Introduction}

Gastric cancer (GC) is the fourth most common type of cancer in the world. The incidence rates for GC are highest

Correspondence to: Professor Wei-Guo Dong, Department of Gastroenterology, Renmin Hospital of Wuhan University, 238 Jiefang Road, Wuhan, Hubei 430060, P.R. China

E-mail:dwg@whu.edu.cn

Key words: ezrin-radixin-moesin-binding phosphoprotein 50, tumor marker; gastric cancer, quantum dot, prognosis, immunochemistry in males from Northeast Asia (Japan, Korea and China) (1), with up to 69 cases per 100,000 individuals per year. The carcinoembryonic antigen, CEA, and the carbohydrate antigens (CA) 19-9 and 72-4, have previously been used as tumor markers (2-3). Specifically, they have been used as reliable markers for monitoring tumor progression and the response to treatments for $\mathrm{GC}$, including chemotherapy and radiation therapy (4). In the majority of patients with CA 19-9- and CA 72-4-positive GC, a decrease in these marker levels is correlated with a positive clinical outcome following successful resection and treatment (5). Consequently, these antigens are not so accurate as tumor progression predictors for GC diagnosis and follow-up. Therefore, it is important to develop additional markers for the screening and follow-up of patients with GC. Considerable efforts have been dedicated to the identification of sensitive and specific markers for GC (6-9). If the clinical predictors that identify patients with a low-risk of cancer recurrence following surgical resection were determined, then low-risk patients would be able to avoid unnecessary post-operative chemotherapy, thus improving their quality of life.

Ezrin-radixin-moesin-binding phosphoprotein 50 (EBP50) is a 358-amino acid protein containing two postsynaptic density-95/disc-large/zonula occludens-1 (PDZ) domains. EBP50 functions as a linker between membrane proteins and the cytoskeleton network and is involved in various types of cancer (10-11). EBP50 is important in cancer progression as it regulates cell proliferation and migration (12). Several studies have observed that EBP50 is a novel marker for various types of cancer, including breast cancer, and that EBP50 is able to predict the clinical behavior of these tumors (11). In a previous study, EBP50 was positively associated with tumor grade, prognosis and the estrogen receptor in the circulatory lymphocytes and breast cancer tissues (13).

EBP50 has been shown to be expressed in gastric parietal cells, as opposed to the mucous epithelium of the stomach which only expresses ezrin (14). However, to the best of our knowledge, there is no available data concerning EBP50 expression in GC. On the basis of data from previous cancer studies, we hypothesized that the EBP50 protein expression level was correlated with the progression of GC. Therefore, quantum dot (QD) and immunohistochemistry (IHC) assays were performed to investigate the prognostic value of EBP50 in $\mathrm{GC}$. 


\section{Materials and methods}

Patients. The present study included $101 \mathrm{GC}$ patients (29 females and 72 males, aged 24-81 years and of Chinese nationality) diagnosed and treated at the General Surgery Department of Renmin Hospital of Wuhan University (Wuhan, China) between 2000 and 2005. Resected tissues were fixed in formalin, then embedded in paraffin. Stage IV GC tissue was unavailable due to a requirement for surgery in the affected patients (Table I). The tumor staging was based on a histopathological analysis and clinical assessment, according to the TNM (tumor-node-metastases) classification. Patients were staged according to the American Joint Committee on Cancer-International Union Against Cancer classification (15). For statistical analysis, the GC patients were divided into two groups, with 47 cancer patients in the stage I-II group and 54 patients in the stage III group. The patients were also subdivided into four groups depending on the degree of gastric wall invasion $\left(\mathrm{T}_{1}, \mathrm{~T}_{2}, \mathrm{~T}_{3}\right.$ and $\left.\mathrm{T}_{4}\right)$ and four other groups depending on the nodal involvement $\left(\mathrm{N}_{0}, \mathrm{~N}_{1}, \mathrm{~N}_{2}\right.$ and $\mathrm{N}_{3}$ ). The number of patients in these analyzed subgroups is shown in Table I. The present study was approved by the Ethics Committee of Wuhan University. Consent was received from all patients and all clinical investigations were performed according to the principles of the Declaration of Helsinki.

IHC analysis. The GC tissues were fixed in $10 \%$ buffered formalin, embedded in paraffin and cut into $4-\mu \mathrm{m}$ sections. The sections were deparaffinized in xylene, rehydrated in a series of descending ethanol concentrations and incubated in $0.03 \%$ hydrogen peroxide for $10 \mathrm{~min}$. Antigen retrieval was performed in $10 \mathrm{mM}$ sodium citrate buffer ( $\mathrm{pH} \mathrm{6.0)}$ for $15 \mathrm{~min}$. The tissue sections were then incubated with an anti-EBP50 antibody (1:800 dilution, PA5-17045; Thermo Scientific, Rockford, IL, USA) at room temperature for $40 \mathrm{~min}$. Following incubation, the specimens were washed with $0.5 \%$ Tween, $0.1 \mathrm{M}$ Tris-base, $0.9 \% \mathrm{NaCl}$, (TBS-T; $\mathrm{pH}$ 7.6) and incubated with peroxidase-labeled polymer at room temperature for $30 \mathrm{~min}$. The samples were then washed with TBS-T buffer and incubated with freshly prepared 3,3'-diaminobenzidine tetrahydrochloride (DAB) and substrate-chromogen buffer at room temperature for $8 \mathrm{~min}$. Immunohistochemical reactions were developed in freshly prepared DAB (DAB kit; Fujian Maixin Biological Technology Ltd., Fujian, China) at room temperature for $8 \mathrm{~min}$, then lightly counterstained with hematoxylin prior to mounting.

QD fluorescence IHC. The specimen treatment by QD-IHC was similar to conventional IHC. The QD-IHC assay was performed according to the manufacturer's instructions (Wuhan Jiayuan Quantum Dot Co., Ltd., Wuhan, China). Antigen retrieval was performed in citric acid (10 mM, pH 6.0) at $95^{\circ} \mathrm{C}$ for $10 \mathrm{~min}$ and the samples were cooled for $30 \mathrm{~min}$. For the antibody binding, the specimens were first incubated in a $2 \%$ bovine serum albumin buffer (Sigma, St. Louis, $\mathrm{MO}, \mathrm{USA})$ at $37^{\circ} \mathrm{C}$ for $30 \mathrm{~min}$ and then at $4^{\circ} \mathrm{C}$ overnight with poly-rabbit anti-EBP50 antibodies (1:800 dilution, PA5-17045; Thermo Scientific, Rockford, IL, USA). The specimens were then washed three times with TBS-T for 5 min each wash and
Table I. Characteristics of the patients with GC.

\begin{tabular}{|c|c|}
\hline Tested group & Number $(\%$ \\
\hline \multicolumn{2}{|l|}{ Age, years } \\
\hline$\leq 50$ & $24(23.8)$ \\
\hline$>50$ & $77(76.2)$ \\
\hline \multicolumn{2}{|l|}{ Gender } \\
\hline Male & $72(71.3)$ \\
\hline Female & $29(28.7)$ \\
\hline \multicolumn{2}{|l|}{ Differentiation } \\
\hline Well & $16(15.8)$ \\
\hline Moderate & 37 (36.6) \\
\hline Undifferentiated & $48(47.6)$ \\
\hline \multicolumn{2}{|l|}{ Tumor size } \\
\hline $\mathrm{T}_{1}$ & $8(7.9)$ \\
\hline $\mathrm{T}_{2}$ & $21(20.8)$ \\
\hline $\mathrm{T}_{3}$ & $55(54.5)$ \\
\hline $\mathrm{T}_{4}$ & $17(16.8)$ \\
\hline \multicolumn{2}{|l|}{ Nodal metastasis } \\
\hline $\mathrm{N}_{0}$ & $33(32.7)$ \\
\hline $\mathrm{N}_{1}$ & $48(47.5)$ \\
\hline $\mathrm{N}_{2}$ & 13 (12.9) \\
\hline $\mathrm{N}_{3}$ & $7(6.9)$ \\
\hline \multicolumn{2}{|l|}{ Stage } \\
\hline I-II & $47(46.1)$ \\
\hline III & $54(53.9)$ \\
\hline
\end{tabular}

$\mathrm{GC}$, gastric cancer.

incubated in biotinylated goat anti-rabbit or anti-mouse IgG (1:100 dilution, Jackson ImmunoResearch, West Grove, PA, USA) at $37^{\circ} \mathrm{C}$ for $30 \mathrm{~min}$.

For the QD conjugation, the antibody-bound specimens were incubated in $2 \% \mathrm{BSA}$ buffer again at $37^{\circ} \mathrm{C}$ for $10 \mathrm{~min}$, then incubated with QDs conjugated to streptavidin (QD-SA; 1:200 dilution in 2\% BSA; Wuhan Jiayuan Quantum Dot Co., Ltd.) at $37^{\circ} \mathrm{C}$ for $30 \mathrm{~min}$, washed three times with TBS-T for 5 min and finally sealed with $90 \%$ glycerin (Sigma).

The QD signal was detected with an Olympus BX51 fluorescence microscope equipped with an Olympus Micro DP 72 camera. The signal was red, target-specific, bright and photostable. The images for each specimen were analyzed using the WuDa Image Analysis System 2003, a multifunctional pathology analysis software package developed by two of the present study authors (16-18). For further quantification and statistical analysis, the numerical calculations for the two key variables in EBP50 detection, the fluorescence intensity and the distribution area, were based on spectral unmixing and the QDs were obtained as the final results.

Scoring of $Q D-I H C$ and $I H C$. Using a 400X objective lens, $\geq 100$ cells were randomly selected and counted from five representative fields of each core by two independent observers blinded to the samples identities. The scoring of the QD-IHC and IHC was based on the percentage of positive 
Table II. Correlations between EBP50 protein expression and the clinical significance of 101 cases of GC.

\begin{tabular}{|c|c|c|c|c|c|c|c|}
\hline \multirow[b]{2}{*}{ Tested group } & \multicolumn{4}{|c|}{ IHC assessment of EBP50 } & \multicolumn{3}{|c|}{ QD score of EBP50 } \\
\hline & Negative & Positive & Total & P-value & Number & $\mathrm{QD}(\mathrm{X} \pm \mathrm{SD})$ & P-value \\
\hline \multicolumn{8}{|l|}{ Age, years } \\
\hline$\leq 50$ & 10 & 14 & 24 & 0.640 & 24 & $11.810 \pm 3.937$ & 0.456 \\
\hline$>50$ & 28 & 49 & 77 & & 77 & $13.250 \pm 3.407$ & \\
\hline \multicolumn{8}{|l|}{ Gender } \\
\hline Male & 21 & 51 & 72 & 0.006 & 72 & $13.477 \pm 6.355$ & 0.933 \\
\hline Female & 17 & 12 & 29 & & 29 & $9.433 \pm 5.687$ & \\
\hline \multicolumn{8}{|l|}{ Differentiation } \\
\hline Well & 6 & 10 & 16 & 0.916 & 16 & $26.446 \pm 6.675$ & 0.148 \\
\hline Moderate & 13 & 24 & 37 & & 37 & $11.633 \pm 4.846$ & \\
\hline Undifferentiated & 19 & 29 & 48 & & 48 & $36.135 \pm 11.039$ & \\
\hline \multicolumn{8}{|l|}{ Tumor size } \\
\hline $\mathrm{T}_{1}$ & 6 & 2 & 8 & 0.020 & 8 & $33.708 \pm 17.851$ & 0.029 \\
\hline $\mathrm{T}_{2}$ & 9 & 12 & 21 & & 21 & $6.548 \pm 4.192$ & \\
\hline $\mathrm{T}_{3}$ & 21 & 34 & 55 & & 55 & $28.210 \pm 7.202$ & \\
\hline $\mathrm{T}_{4}$ & 2 & 15 & 17 & & 17 & $41.935 \pm 8.167$ & \\
\hline \multicolumn{8}{|l|}{ Nodal metastasis } \\
\hline $\mathrm{N}_{0}$ & 18 & 15 & 33 & 0.099 & 33 & $28.747 \pm 4.021$ & 0.717 \\
\hline $\mathrm{N}_{1}$ & 15 & 33 & 48 & & 48 & $20.057 \pm 8.102$ & \\
\hline $\mathrm{N}_{2}$ & 3 & 10 & 13 & & 13 & $29.271 \pm 10.734$ & \\
\hline $\mathrm{N}_{3}$ & 2 & 5 & 7 & & 7 & $25.645 \pm 15.087$ & \\
\hline \multicolumn{8}{|l|}{ Stage } \\
\hline I-II & 35 & 12 & 47 & 0.537 & 47 & $28.467 \pm 9.127$ & 0.918 \\
\hline III & 43 & 11 & 54 & & 54 & $25.941 \pm 15.540$ & \\
\hline
\end{tabular}

EBP50, ezrin-radixin-moesin-binding phosphoprotein 50; GC, gastric cancer; QD, quantum dot; IHC, immunohistochemistry.

levels as follows: No staining or weak staining in $<10 \%$ of the tumor cells ( 0 ); weak staining in $>10 \%$ of the tumor cells $(1+)$; complete staining of the membrane with weak or moderate intensity in $>10 \%$ of the tumor cells $(2+)$; and marked staining in $>10 \%$ of the tumor cells (3+) (19).

Statistical analysis. All statistical analysis was performed using SPSS software, Version 13.0 (SPSS Inc., Chicago, IL, USA). The differences between the two groups, based on the clinicopathological factors, were statistically analyzed using the Student's t-test and the Chi-squared test. The survival rates were calculated according to the Kaplan-Meier method, using a log-rank test. $\mathrm{P}<0.05$ was considered to indicate a statistically significant difference.

\section{Results}

Detection of EBP50 expression by IHC. The EBP50 protein was overexpressed in the majority of gastric carcinoma tissues $(63 / 101,62.4 \%)$, of these, 37 tissue samples $(36.6 \%)$ were scored as $1+, 16(15.8 \%)$ were scored as $2+$ and $10(9.9 \%)$ were scored as $3+$ (Fig. 1). The expression of EBP50 detected by IHC was associated with tumor size and the male gender $(\mathrm{P}<0.05)$. The expression of EBP50 in the patients with GC increased with the tumor stage and was highest in the male patients (Table II).

Detection of EBP50 expression by QD analysis. As shown in Fig. 2, the bright-red QD fluorescence specifically labeled tumor cells without nonspecific binding. The green background was from tissue autofluorescence. The score of the QDs was associated with the tumor size of the gastric carcinoma $(\mathrm{P}<0.05$; Table II).

Survival analysis. A survival analysis was performed on 101 patients who had survived for more than one month post-surgery. A total of 38 patients succumbed to GC within 124 months, while 63 had survived survived to January 1, 2012. The survival curves created according to the IHC and QD results of the EBP50 expression are shown in Fig. 3. The association between the expression of EBP50 and the mean survival rates was assessed by the Kaplan-Meier method. The survival rates with overexpression of EBP50 was higher than the negative one, however as $\mathrm{P}>0.05$, there was no association between the survival rates and expression of EBP50. (IHC, 50.5 vs. 58.1 months, P>0.05; QD, 55.4 vs. 63.2 months, P>0.05; Fig. 3 ). 

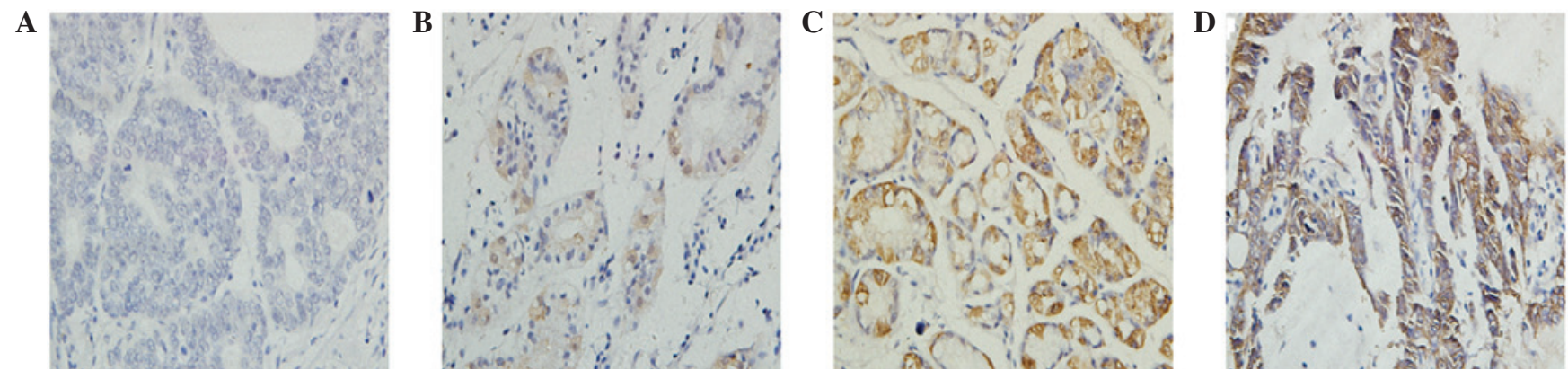

Figure 1. Immunohistochemistry showing EBP50 staining in GC. Sample of (A) negative EBP50 protein expression, (B) a positive case scoring 1+; (C) a positive case scoring 2+, and (D) a positive case scoring 3+ [magnification, x400; objective lens; Streptomyces avidin-peroxidase connection (SP) method]. EBP50, ezrin-radixin-moesin-binding phosphoprotein 50; GC, gastric cancer.
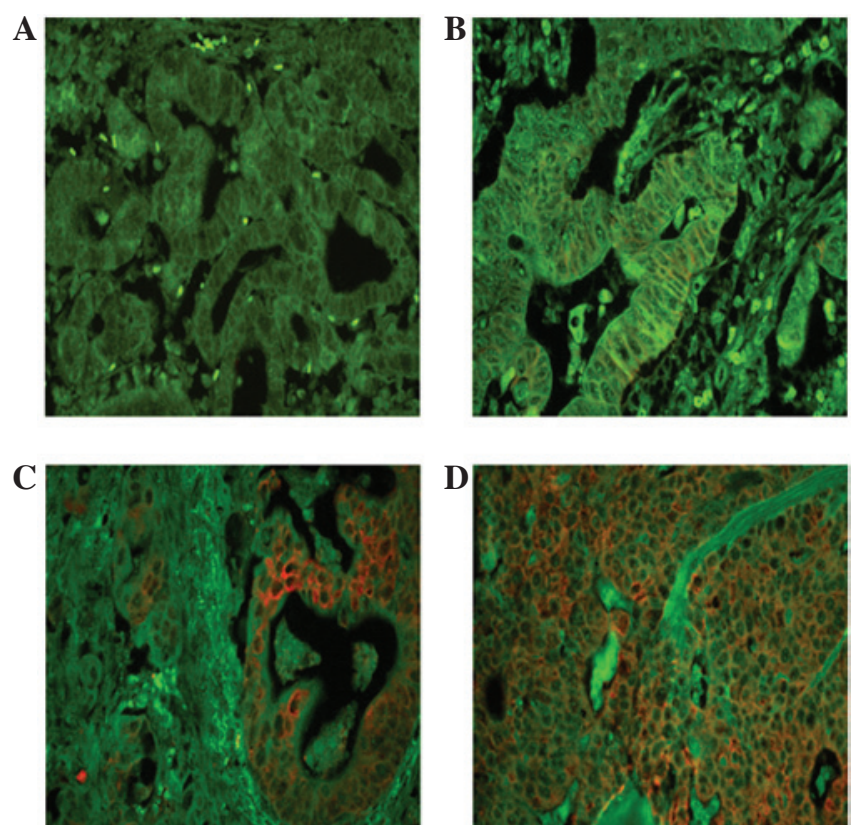

Figure 2. Analysis of EBP50 in advanced gastric cancer using quantum dots (QDs). (A) Sample negative for EBP50 protein expression; (B) a positive case scoring $1+$; (C) a positive case scoring $2+$; and (D) a positive case scoring $3+$ (magnification, x400; objective lens). EBP50, ezrin-radixin-moesin-binding phosphoprotein 50 .

\section{Discussion}

EBP50 may play an essential role in carcinogenesis, including that of breast cancer, colorectal cancer and hepatocellar carcinoma. The present study demonstrated that EBP50 is overexpressed in GC and that it is a novel marker of GC, as observed in previous studies (20-22). The expression of EBP50 was also observed to be correlated with the male gender and the tumor stage. In the present study, the bright red QD fluorescence specifically labeled the GC cell membranes without non-specific binding. Previous studies have demonstrated that EBP50 is important in cancer cell proliferation, invasion and metastasis. Therefore, we hypothesized that the EBP50 protein expression level may be correlated with the prognosis of GC. However, no significant correlation was observed between the expression of EBP50 and the overall survival rate of the GC patients. The observable differences in these studies may be a result of the usage of varying antibodies and scoring systems or, alternatively, may reflect the heterogeneity of GC between
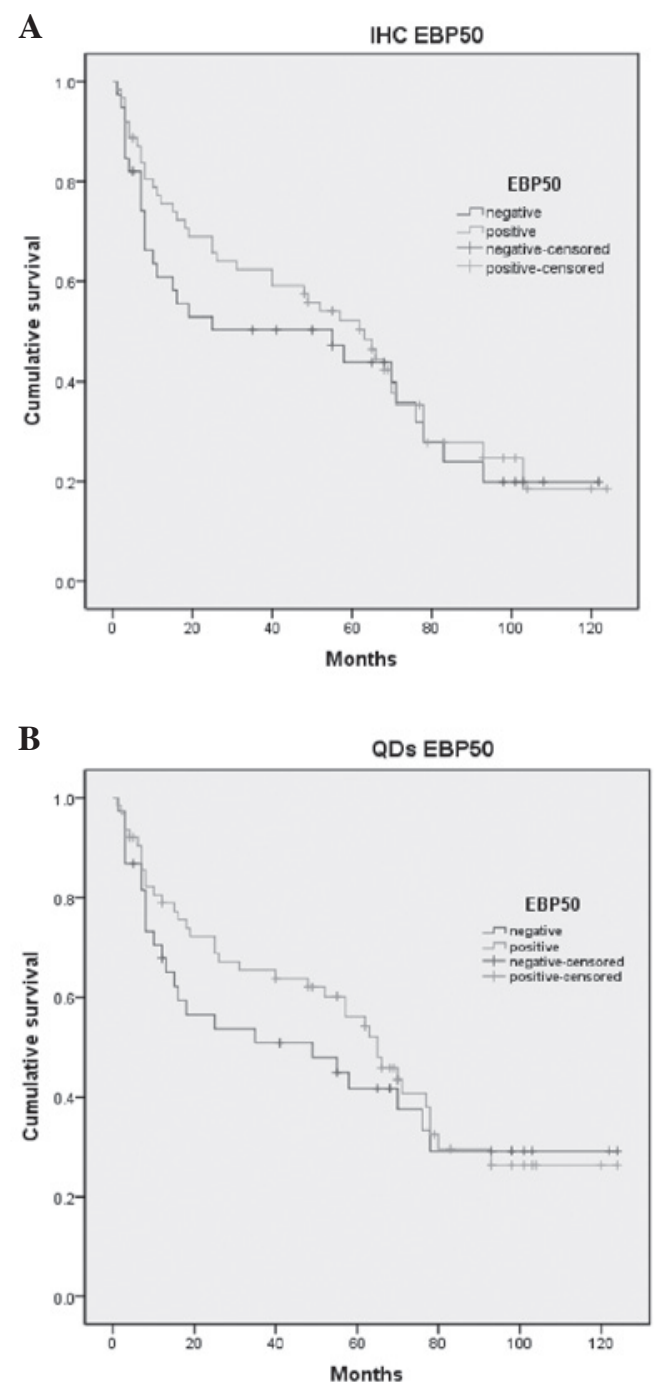

Figure 3. Kaplan-Meier plot for overall survival in 101 patients with advanced GC. The EBP50 protein expression was assessed using (A) IHC and (B) quantum dots (QDs). EBP50, ezrin-radixin-moesin-binding phosphoprotein 50; IHC, immunohistochemistry; GC, gastric cancer.

the various ethnicities. Gastric tumorigenesis is a multistep process that is initiated by benign and atypical hyperproliferation, is established as in situ carcinoma, progresses into invasive carcinoma and culminates in metastatic disease (1,20-21). However, the progression from in situ to invasive carcinoma is 
poorly understood. It is important to identify additional promising tumor markers to improve screening strategies for GC. In the present study, EBP50 immunoreactivity was significantly associated with the male gender and tumor invasion (T stage). These results suggest that EBP50 expression is associated with several malignant clinicopathological features of GC, although it is not a valuable predictor for the prognosis of GC patients.

Immunofluorescence labeling is a standard technique that is widely used in the biomedical field for the detection of biological macromolecules in tissue sections. However, at present, the available fluorescent labels are not stable and become irreversibly photobleached under high-intensity illumination. We used a validated QD-IHC protocol for quantifying EBP50 expression in formalin-fixed, paraffinembedded specimens, which provided an accurate, sensitive and convenient approach. In the present study, 605-nm QD-SA conjugated probes were used for the detection of EBP50 expression. These probes have several advantages that are prerequisites for clinical application $(22,23)$. First, the probes are more stable than QDs conjugated to antibodies. Second, the biotin-avidin staining system is commonly used in molecular pathology and is highly sensitive, therefore, QD-SA probes may be incorporated into the current detection system easily and conveniently.

In conclusion, the overexpression of EBP50 is correlated with the male gender and with tumor size in GC, but not with the patient survival time. Additionally, QD-IHC is as good as IHC for the detection of tumor markers when considering the accuracy of detecting the protein location, the photostability and the duration of the fluorescence lifetime.

\section{References}

1. Hartgrink HH, Jansen EP, van Grieken NC and van de Velde CJ: Gastric cancer. Lancet 374: 477-490, 2009.

2. Kodera Y, Yamamura Y, Torii A, Uesaka K, Hirai T, Yasui K, et al: The prognostic value of preoperative serum levels of CEA and CA19-9 in patients with gastric cancer. Am J Gastroenterol 91: 49-53, 1996.

3. Tocchi A, Costa G, Lepre L, Liotta G, Mazzoni G, Cianetti A and Vannini P: The role of serum and gastric juice levels of carcinoembryonic antigen, CA19.9 and CA72.4 in patients with gastric cancer. J Cancer Res Clin Oncol 124: 450-455, 1998.

4. Hummel R, Hussey DJ and Haier J: MicroRNAs: predictors and modifiers of chemo- and radiotherapy in different tumour types. Eur J Cancer 46: 298-311, 2010.

5. Yamao T, Kai S, Kazami A, Koizumi K, Handa T, Takemoto N and Maruyama M: Tumor markers CEA, CA19-9 and CA125 in monitoring of response to systemic chemotherapy in patients with advanced gastric cancer. Jpn J Clin Oncol 29: 550-555,1999.

6. Macdonald JS, Smalley SR, Benedetti J, Hundahl SA, Estes NC, Stemmermann GN, et al: Chemoradiotherapy after surgery compared with surgery alone for adenocarcinoma of the stomach or gastroesophageal junction. N Engl J Med 345: 725-730, 2001.

7. Ichikawa D, Koike H, Ikoma H, Ikoma D, Tani N, Otsuji E, et al Detection of aberrant methylation as a tumor marker in serum of patients with gastric cancer. Anticancer Res 24: 2477-2481, 2004.
8. Tani N, Ichikawa D, Ikoma D, Tomita H, Sai S, Ikoma H, et al: Circulating cell-free mRNA in plasma as a tumor marker for patients with primary and recurrent gastric cancer. Anticancer Res 27: 1207-1212, 2007.

9. Li N, Zhang J, Liang Y, Shao J, Peng F, Sun M, et al: A controversial tumor marker: is SM22 a proper biomarker for gastric cancer cells? J Proteome Res 6: 3304-3312, 2007.

10. Shibata T, Chuma M, Kokubu A, Sakamoto M and Hirohashi S: EBP50, a beta-catenin-associating protein, enhances Wnt signaling and is over-expressed in hepatocellular carcinoma. Hepatology 38: 178-186, 2003.

11. Song J, Bai J, Yang W, Gabrielson EW, Chan DW and Zhang Z: Expression and clinicopathological significance of oestrogen-responsive ezrin-radixin-moesin-binding phosphoprotein 50 in breast cancer. Histopathology 51: 40-53, 2007.

12. Zheng JF, Sun LC, Liu H, Huang Y, Li Y and He J: EBP50 exerts tumor suppressor activity by promoting cell apoptosis and retarding extracellular signal-regulated kinase activity. Amino Acids 38: 1261-1268, 2010.

13. Bellizzi A, Mangia A, Malfettone A, Cardone RA, Simone G, Reshkin SJ and Paradiso A: $\mathrm{Na}+\mathrm{H}+$ exchanger regulatory factor 1 expression levels in blood and tissue predict breast tumour clinical behaviour. Histopathology 58: 1086-1095, 2011.

14. Ingraffea J, Reczek D and Bretscher A: Distinct cell type-specific expression of scaffolding proteins EBP50 and E3KARP: EBP50 is generally expressed with ezrin in specific epithelia, whereas E3KARP is not. Eur J Cell Biol 81: 61-68, 2002.

15. Sobin, LH and Fleming, ID: TNM Classification of Malignant Tumors, fifth edition (1997). Union Internationale Contre le Cancer and the American Joint Committee on Cancer. Cancer 80: 1803-1804, 1997.

16. Hu Y, Wu Q, Liu S, Wei L, Chen X, Yan Z, et al: Study of rice pollen grains by multispectral imaging microscopy. Microsc Res Tech 68: 335-346, 2005.

17. Guo N, Zeng L and Wu Q: A method based on multispectral imaging technique for white blood cell segmentation. Comput Biol Med 37: 70-76, 2007.

18. Dickinson ME, Bearman G, Tille S, Lansford R and Fraser SE: Multi-spectral imaging and linear unmixing add a whole new dimension to laser scanning fluorescence microscopy. Biotechniques 31: 1272, 1274-1276, 1278, 2001.

19. Chen H, Xue J, Zhang Y, Zhu X, Gao J and Yu B: Comparison of quantum dots immunofluorescence histochemistry and conventional immunohistochemistry for the detection of caveolin-1 and PCNA in the lung cancer tissue microarray. J Mol Histol 40: 261-268, 2009.

20. Yao W, Feng D, Bian W, et al: EBP50 inhibits EGF-induced breast cancer cell proliferation by blocking EGFR phosphorylation. Amino Acids 43: 2027-2035, 2012.

21. Kislin KL, McDonough WS, Eschbacher JM, et al: NHERF-1: modulator of glioblastoma cell migration and invasion. Neoplasia 11: 377-387, 2009.

22. Malfettone A, Saponaro C, Paradiso A, et al: Peritumoral vascular invasion and NHERF1 expression define an immunophenotype of grade 2 invasive breast cancer associated with poor prognosis. BMC Cancer 12: 106, 2012.

23. Bornschein J and Malfertheiner P: Gastric carcinogenesis. Langenbecks Arch Surg 396: 729-742, 2011.

24. Kawachi T, Kurisu M, Numanyu N, Sasajima K and Sano T: Precancerous changes in the stomach. Cancer Res 36: 2673-2677, 1976.

25. Muthu MS, Kulkarni SA, Raju A and Feng SS: Theranostic liposomes of TPGS coating for targeted co-delivery of docetaxel and quantum dots. Biomaterials 33: 3494-3501, 2012.

26. Shao L, Gao Y and Yan F: Semiconductor quantum dots for biomedicial applications. Sensors (Basel) 11: 11736-11751, 2011. 